\title{
Effectiveness of Controlled Release Chlorhexidine Chip as an Adjunctive to Scaling and Root Planning for the Treatment of Chronic Periodontitis
}

\author{
Rashmi Gupta ${ }^{1}$, Preetinder Singh ${ }^{2}$, Yashpaul Dev ${ }^{3}$, Shipra Sardana ${ }^{4}$, Kirti Rathee ${ }^{5}$, Mallika Sethi ${ }^{6}$
}

\begin{abstract}
Background: Chlorhexidine is considered one of the options for the management of periodontal diseases. This present study was conducted to assess the usefulness of controlled-release chlorhexidine chip in addition to scaling and root planning (SRP) while comparing with SRP alone in the treatment of chronic periodontitis clinically, microbiologically and radiographically.

Materials and methods: This study included 20 patients age ranged from 30 to 55 years with chronic periodontitis. A total of 40 sites were selected. Two bilateral pockets with probing depth 4-6 mm were put in test and control groups in all subjects. SRP plus Periochip ${ }^{\mathrm{TM}}$ was used in the test group and SRP alone in the control group. In all subjects, all the analysis such as clinical parameters, the radiographic and anaerobic culture of $P$. gingivalis, T. denticola, and T. forsythia were performed and compared in both groups at baseline and 3 months interval.

Results: There was a significant improvement in all clinical and radiographic parameters in the test group in comparison to control group. Total colony counts also reduced significantly. At baseline, Tf was recovered from 15 test group sites and 16 control group sites, $P g$ from 14 test group and 14 control group sites, Td from 16 test group and 15 control group sites. At 3 months, Tf was recovered from 4 test group and 10 control group sites, $\mathrm{Pg}$ from 3 test group and 9 control group sites, $T d$ from 4 test group and 8 control group site.

Conclusion: Periochip ${ }^{\mathrm{TM}}$ placement as an adjunct to SRP, showed promising results when compared to SRP alone.

Clinical significance: Periochip is considered the best treatment option in the treatment of chronic periodontitis.

Keywords: Chlorhexidine, Periochip, Scaling and root planning.

The Journal of Contemporary Dental Practice (2019): 10.5005/jp-journals-10024-2679
\end{abstract}

\section{INTRODUCTION}

Chronic periodontitis leads to inflammation in supporting tissues of teeth. There is considerable loss of attachment and bone. It results in the formation of periodontal pocket followed by the recession of the gingiva. ${ }^{1}$ Porphyromonas gingivalis $(\mathrm{Pg})$, Aggregatibacter actinomycetemcomitans ( $\mathrm{Aa})$, Prevotella intermedia $(\mathrm{Pi})$ and Tannerella forsythia, etc. are among various subgingival microbial species whose elevated proportions shows destructive periodontal disease activity. ${ }^{2-5}$

To establish the healthy periodontal tissues, it is crucial to keep the pathogenic microflora of the pocket suppressed ${ }^{6,7}$ SRP is the preferred technique that effectively decreases the microbial load, but within 60 days there may be recolonization as early as after SRP. $^{8-10}$ The use of systemic and topical antimicrobial agents has been considerably increased due to the awareness that periodontal disease is not just an overgrowth of bacteria, but also a shift in bacterial species. ${ }^{11}$

PerioChip ${ }^{\mathrm{TM}}$ is a controlled-release drug delivery system that delivers chlorhexidine in the periodontal pockets. It is approved by the FDA. It is locally applied antiseptic which can be used as an adjunct to SRP procedures for the lessening of the depth of probing pocket. ${ }^{12}$ PerioChip ${ }^{\mathrm{TM}}$ consisted of $2.5 \mathrm{mg}$ of chlorhexidine digluconate in a biodegradable matrix of gelatin and glutaraldehyde which releases $40 \%$ of chlorhexidine within the first 2 hours and the rest over the one week treatment period. The present study was conducted to assess the usefulness of controlledrelease chlorhexidine chip in addition to SRP while comparing with SRP alone in the treatment of chronic periodontitis clinically, microbiologically and radiographically.
${ }^{1-5}$ Department of Periodontology, Swami Devi Dyal Hospital and Dental College, Panchkula, Haryana, India

${ }^{6}$ Department of Periodontics, ITS Centre for Dental Studies and Research, Ghaziabad, Uttar Pradesh, India

Corresponding Author: Rashmi Gupta, Department of Periodontology, Swami Devi Dyal Hospital and Dental College, Panchkula, Haryana, India, Phone: +91 9501544877, e-mail: drrashmigupta@gmail.com

How to cite this article: Gupta R, Singh P, Dev Y, et al. Effectiveness of Controlled Release Chlorhexidine Chip as an Adjunctive to Scaling and Root Planning for the Treatment of Chronic Periodontitis. J Contemp Dent Pract 2019;20(12):1402-1405.

Source of support: Nil

Conflict of interest: None

\section{Materials and Methods}

This study was conducted in the Department of Periodontology and Implantology, Swami Devi Dyal Hospital and Dental College, Barwala (Panchkula) Haryana, India. Ethical approval for the study was obtained from the ethical committee of the institution. All were informed regarding the study, and written consent was obtained before starting the study.

The following inclusion and exclusion criteria were used:

Inclusion criteria included subjects in the age group of 30-55 of both the genders, subjects with probing pocket depth $\geq 4$ to $6 \mathrm{~mm}$ and subjects having at least 20 natural teeth in their mouth. Exclusion criteria included pregnancy and lactating mothers, 
subjects allergic to chlorhexidine, patients with a history of smoking, the presence of overhanging restorations and subjects who have received antibiotics or any other form of periodontal treatment in previous 6 months.

A split-mouth study of twenty subjects having generalized chronic periodontitis with a total number of 40 posterior sites was performed. These sites were divided into group I which was control site where only scaling and root planning was done and group II which was test site where PerioChip. ${ }^{\mathrm{TM}}$ was used in addition to scaling and root planning.

Stents were made on patient casts with the help of self-cure resin. Grooves were prepared on the stent with a bur at the involved site so as to measure clinical attachment level (CAL) and the probing depth of the involved sites with a fixed reference point (FRP) with respect to every site. This was designated as the relative attachment level (RAL).

A total of 80 samples were collected from 40 posterior sites, 20 samples from the control sites (before SRP), 20 samples from test site (before scaling and root planning), 20 samples from control site (after 3 months) and 20 samples from test sites (after 3 months). A sample of the subgingival plaque was collected around the teeth with the help of an area specific curette. Immediately after removal, plaque samples were transferred in the tube containing TRIS-EDTA buffer to the Department of Molecular Biology and Immunology on the same day for nucleic acid extraction, PCR (polymerase chain reaction), detection of the amplified products-gel electrophoresis.

Before starting any treatment, all clinical parameters, i.e., plaque index (PI) given by Silness and Loe, gingival index, probing depth (PD) (measured at four sites around the selected tooth) and relative attachment level (RAL) (measured at four sites around selected tooth) were measured. Radiographic parameters were also recorded for all subjects. The samples of subgingival plaque were also taken from both sides of both groups.

Primer pairs were engaged in a single tube amplification process which was specific to each organism. The selectivity of PCR results from the use of primers that are complementary to the DNA region targeted for amplification under specific thermal cycling conditions. Bacterial identification was completed with this technique that may be helpful in the detection of microorganisms of different species.

\section{Results}

Table 1 shows that at baseline the difference in gingival score was a nonsignificant but significant improvement was seen in the test group after 3 months $(p=0.049)$. Figure 1 shows that at baseline the difference in RAL was a nonsignificant but significant improvement was seen in the test group after 3 months as compared to the control group (0.008). Table 2 shows the intergroup comparison of colonies of $T$. Forsythia which was nonsignificant ( $p=0.705)$. At 3 months control had 10 colonies and test group had only 4 colonies $(p=0.047)$. Table 3 shows that at baseline control had 14 colonies and test group had 16 colonies ( $p=0.465$ ) showing the nonsignificant difference in the beginning. At 3 months, $p$ value came out to be 0.168 showing

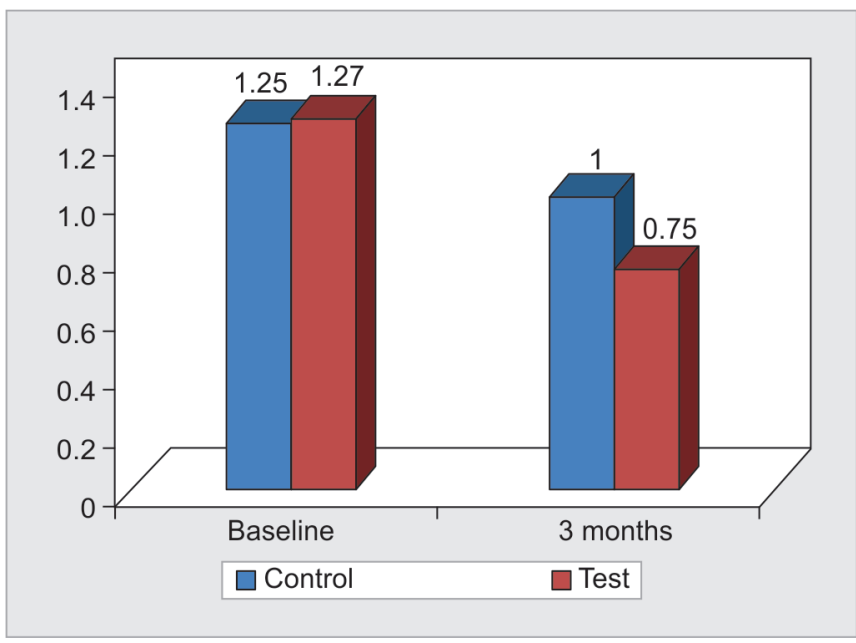

Fig. 1: Intergroup comparison of gingival index

the still nonsignificant difference. Table 4 shows the nonsignificant difference in the radiographic parameter for crestal bone height in both test and control groups at baseline. At 3 months there was nonsignificant $(p>0.05)$ between control and test groups. Results were subjected to statistical analysis. A $p$ value less than 0.05 was considered significant.

\section{Discussion}

Initially, facultative or strict aerobes can lead to orodental infections which shift to anaerobic species at later stages of oral infections. ${ }^{13}$ SRP is an effective means of treating and controlling periodontitis. However, the ability of the therapist to gain access to deep pockets or furcations often results in a substantial variation in its effectiveness. Consequently, this has led to the adjunctive use of antimicrobials. $^{14}$

Local delivery systems consisted of antiseptic or antibiotic drugs allow therapeutic agents to be targeted to diseased sites with minimal systemic effects. This makes us aware of the unwanted use of antibiotics in patients at higher levels that lead to bacterial resistance. Several subgingival drug delivery systems, such as tetracycline fibers, minocycline, doxycycline gel, metronidazole gel, and a chlorhexidine gluconate chip, have been developed and used in controlled clinical trials. ${ }^{15}$

Compared to antibiotics, antiseptic agents have a much broader spectrum of antibacterial activity due to multiple intracellular targets which reduce the possibility of development of bacterial resistance. ${ }^{16}$ The most widely used and studied antiseptic in oral diseases is chlorhexidine. Chlorhexidine shows a high affinity towards bacteria because of the interaction between the positively charged parts of the molecule and the negatively charged phosphate groups of lipopolysaccharides on the bacterial cell walls. The main advantages of chlorhexidine application during SRP or surgical periodontal treatment include better healing of wound and control of plaque. ${ }^{17}$ However, despite the advantages, the

Table 1: Intergroup comparison of plaque score

\begin{tabular}{lllllll}
\hline & Control & Test & & \multicolumn{3}{c}{ Comparison using Mann-Whitney test } \\
\cline { 2 - 3 } \cline { 6 - 8 } & Mean $\pm S D$ & Mean $\pm S D$ & & $Z$ & $p$ value & Sig. \\
\hline Baseline & $0.90 \pm 0.22$ & $0.92 \pm 0.23$ & & -1.146 & 0.252 & NS \\
3 months & $0.72 \pm 0.23$ & $0.52 \pm 0.23$ & & -2.727 & 0.010 & $\mathrm{~S}$ \\
\hline
\end{tabular}


Effectiveness of Controlled Release Chlorhexidine Chip as an Adjunctive to Scaling and Root Planning

Table 2: Intergroup comparison of probing depth

\begin{tabular}{lllllll}
\hline & Control & Test & & \multicolumn{3}{c}{ Comparison using Mann-Whitney test } \\
\cline { 2 - 3 } \cline { 6 - 8 } Duration & Mean $\pm S D$ & Mean $\pm S D$ & & & Z value & Sig. \\
\hline Baseline & $6.10 \pm 1.51$ & $5.35 \pm 0.58$ & & -1.586 & 0.113 & NS \\
3 months & $5.35 \pm 0.81$ & $4.50 \pm 1.05$ & & -2.498 & 0.012 & S \\
\hline
\end{tabular}

Table 3: Intergroup comparison of RAL

\begin{tabular}{lllllll}
\hline & Control & Test & & \multicolumn{3}{c}{ Comparison using Mann-Whitney test } \\
\cline { 2 - 3 } \cline { 6 - 7 } Duration & Mean $\pm S D$ & Mean $\pm S D$ & & $Z$ & Z value & Sig. \\
\hline Baseline & $11.35 \pm 1.18$ & $11.85 \pm 0.93$ & & -1.309 & 0.191 & NS \\
3 months & $9.70 \pm 1.03$ & $10.90 \pm 1.41$ & & -2.635 & 0.008 & S \\
\hline
\end{tabular}

Table 4: Intergroup comparison of RF

\begin{tabular}{lllllll}
\hline & Control & Test & & \multicolumn{3}{c}{ Comparison using Mann-Whitney test } \\
\cline { 2 - 3 } \cline { 6 - 8 } Duration & Mean $\pm S D$ & Mean $\pm S D$ & & & Z value & Sig. \\
\hline Baseline & $3.00 \pm 0.97$ & $2.80 \pm 1.47$ & & -0.648 & 0.517 & NS \\
3 months & $2.85 \pm 0.93$ & $2.55 \pm 1.23$ & & -0.856 & 0.392 & NS \\
\hline
\end{tabular}

application may result in staining of the teeth, taste disturbances and an increase of calculus formation. ${ }^{18}$

When the PerioChip is split, chlorhexidine is liberated gradually in gingival crevicular fluid (GCF) at the level of $125 \mu \mathrm{g} / \mathrm{mL}$ for 7-10 days. After 2-4 days the concentration of drug reaches $1,300 \mu \mathrm{g} / \mathrm{mL}$ and then decreases but remains above $125 \mu \mathrm{g} / \mathrm{mL}$ in the first 7 days. ${ }^{19}$ Albeit successful results have been described for use of PerioChip ${ }^{\mathrm{TM}}$, there are very few studies that include clinical, microbiological analysis, and radiographic parameters in a single study. It is worthwhile for patients and clinicians to know whether the use of PerioChip ${ }^{\mathrm{TM}}$ represents a predictive, reliable and effective therapy in periodontitis. ${ }^{20}$ Hence, the present study aimed to evaluate the effectiveness of a controlled-release chlorhexidine chip as adjunctive therapy to scaling and root planing in the treatment of chronic periodontitis.

Many studies have shown that as the depth increases to $5 \mathrm{~mm}$, the complete access to the subgingival area with hand instruments decreases and the efficacy of scaling and root planning becomes progressively lesser effective as the instrument is unable to reach the base of the pocket. This resulted in the use of irrigants or systemic antibiotics as antibacterial agents to conquer the incomplete scope of conventional treatment. ${ }^{21}$

Goodson et al. ${ }^{21}$ in their study explained the purpose and use of controlled delivery of drugs in the treatment of periodontitis. He postulated that with this therapy, the drug may reach to the base of the periodontal pocket and is maintained for an adequate duration for the antimicrobial effect to occur. Soskolne et al., ${ }^{22}$ Stabholz et al. ${ }^{23}$ in their studies suggested that local delivery of drug may last up to 11 weeks after administration which corresponds to the typical recall interval for periodontal patients.

When we compared the results of plaque scores of our study with the results of Soskolne et al., ${ }^{22}$ Stabholz et al., ${ }^{23}$ Soskolne et al., ${ }^{22}$ and Marjorie et al. ${ }^{24}$ our results were found to be in accordance with the latter who observed a change in plaque score from baseline to 3 months. Findings of the present study are also in partial accordance with Rodrigues et al., ${ }^{15}$ as they also found a reduction in plaque score in both treatment groups which was statistically significant.
According to a study conducted by Grover et al., ${ }^{25}$ there was bone gain from 1 to $2 \mathrm{~mm}$, at 3-month intervals at $21 \%$ of the sites in patients of group I, i.e., control group. There was an only bone loss in $5 \%$ of the sites whereas $74 \%$ of the sites maintained the same bone level. In patients of group II, i.e., test group, there was bone gain ranging from 1 to $2 \mathrm{~mm}$ at $47 \%$ of the sites whereas none of the sites showed a bone loss and $53 \%$ of the sites maintained the bone level. The difference was found to be statistically highly significant in both groups. The small patient size is the limitation of the study. Studies at a larger scale are required to substantiate the results obtained in this study.

\section{Conclusion}

Authors found that PerioChip showed significant reductions in the numbers of the putative periodontopathic organisms including red complex microbes like Porphyromonas gingivalis (Bacteroides) and also on others like Prevotella intermedia (Bacteroides), Bacteroides forsythus and Campylobacter rectus (Wolinella recta) after placement of the chip.

\section{Clinical Significance}

Periochip is considered the best treatment option in the treatment of chronic periodontitis.

\section{References}

1. Periodontitis. Glossary of Periodontal Terms: American Academy of Periodontology; 2001. p. 40.

2. Rams TE, Slots J. Local delivery of antimicrobial agents in the periodontal pocket. Periodontal 2000 1996;10:139-159. DOI: 10.1111/ j.1600-0757.1996.tb00072.x.

3. Marsh PD. Dental plaque: Biological significance of a biofilm and community lifestyle. J Clin Periodontol 2005;32:7-15. DOI: 10.1111/j.1600-051X.2005.00790.x.

4. Slots J, Rams TE, Listgarten MA. Yeasts, Enteric rods and pseudomonas in the subgingival flora of severe adult periodontitis. Oral Microbial Immunol 1988;3:47-52. DOI: 10.1111/j.1399-302X.1988.tb00080.x.

5. Listgarten MA. The structure of dental plaque. Periodontol 2000 1994;5:52-65. DOI: 10.1111/j.1600-0757.1994.tb00018.x. 
6. Bruce LP, William FA. Treatment of gingivitis and periodontitis (position paper). J Periodontol 1997;68:1246-1253.

7. Cobb CM. Non surgical pocket therapy-mechanical. Ann Periodontal 1996;1:443-490. DOI: 10.1902/annals.1996.1.1.443.

8. Donald A. Consensus report: Non surgical pocket therapy: Pharmacotherapeutics and dental occlusion. Ann Periodontol 1996;1:581-588. DOI: 10.1902/annals.1996.1.1.581.

9. Mandel ID. Chemotherapeutic agents for controlling plaque and gingivitis. J Clin Periodontol 1988;15:488-498. DOI: 10.1111/j.1600051X.1988.tb01020.x.

10. Stabholz A, Sela MN, Friedman M, et al. Clinical and microbiological effects of sustained release chlorhexidine in periodontal pockets. J Clin Periodontol 1986;13:783-788. DOI: 10.1111/j.1600-051X.1986. tb00882.x.

11. Slots J. Selection of antimicrobial agents in periodontal therapy. J Periodontal Res 2002;37:389-398. DOI: 10.1034/j.16000765.2002.00004.x.

12. Ryan ME. Nonsurgical approaches for the treatment of periodontal diseases. Dent Clin North Am 2005;49:611-636. DOI: 10.1016/ j.cden.2005.03.010.

13. Chaturvedi TP, Srivastava R, Srivastava AK, et al. Evaluation of metronidazole nanofibers in patients with chronic periodontitis: a clinical study. Int J Pharm Investig 2012;2:213-217. DOI: 10.4103/2230973X.107007.

14. Cosyn J,Wyn I. A Systematic Review on the Effects of the Chlorhexidine Chip When Used as an Adjunct to Scaling and Root Planing in the Treatment of Chronic Periodontitis. J Periodontol 2006;77:257-264. DOI: 10.1902/jop.2006.050216.

15. Rodrigues IFG, Machion L, Casati MZ, et al. Clinical Evaluation of the Use of Locally Delivered Chlorhexidine in Periodontal Maintenance Therapy. J Periodontol 2007;78:624-628. DOI: 10.1902/jop.2007. 060317.

16. Attin R, llse A, Werner C, et al. Antimicrobial effectiveness of a highly concentrated chlorhexidine varnish treatment in teenagers with fixed orthodontic appliances. Angle Orthod 2006;76:1022-1027. DOI 10.2319/070605-218.

17. Imfeld T. Chlorhexidine containing chewing gum. Clinical documentation. Schweiz Monnatsschr Zahnmed 2006;116:476-483.

18. Zanatta FB, Antoniazzi RD, Rosig CK. Staining and calculus formation after $0.12 \%$ chlorhexidine rinses in plaque-free and plaque covered surfaces: a randomized trial. J Appl Oral Sci 2010;18:515-521. DOI: 10.1590/S1678-77572010000500015.

19. Soskolne WA, Chajek T, Flashner M, et al. An in vivo study of the chlorhexidine release profile of the PerioChip ${ }^{\mathrm{TM}}$ in the gingival crevicular fluid, plasma and urine. J Clin Periodontol 1998;25: 1017-1021. DOI: 10.1111/j.1600-051X.1998.tb02407.x.

20. Marjorie JK, Bray KS, Sebestian G, et al. Adjunctive use of a subgingival release chlorhexidine chip reducing probing depth and improves attachment level compared with scaling and root planing alone. J Periodontol 1998;69:989-997. DOI: 10.1902/jop.1998.69.9.989.

21. Goodson JM, Offenbacher S, Farr DH, et al. Periodontol disease treated by local drug delivery. J Periodontol 1985;56:265-272. DOI: 10.1902/ jop.1985.56.5.265.

22. Soskolne A, Gololmb G, Micheal F, et al. New sustained dosage form of chlorhexidine for dental use. L Periodontol Res 1983;18:330-336. DOI: 10.1111/j.1600-0765.1983.tb00368.x.

23. Stabholz A, Sela M, Friedman M, et al. Clinical and microbiological effect of sustained release chlorhexidine in periodontal pocket. J Clin Periodontol 1986;13:787-788. DOI: 10.1111/j.1600-051X.1986. tb00882.x.

24. Marjorie JK, Kent PG, Thomas WW, et al. Use of a biodegradable chlorhexidine chip in the treatment of adult periodontitis: clinical and radiographic findings. J Periodontol 2000;71:256-261. DOI: 10.1902/ jop.2000.71.2.256

25. Grover V, Kapoor A, Malhotra R, et al. To assess the effectiveness of a chlorhexidine chip in the treatment of chronic periodontitis: a clinical and radiographic study. J Indian sos Periodontal 2011;15:139-146. DOI: 10.4103/0972-124X.84383. 